\title{
Design of Intelligent Drawer Control System Based on Speech Recognition Technology
}

\author{
Wang Shaogang ${ }^{1, a}$, Yan Yinfa ${ }^{1}$, Song Zhanhua ${ }^{1}$, Song Hualu ${ }^{1}$, Li Fa-de ${ }^{* 2, b}$ \\ 1. Mechanical and Electronic Engineering College, Shandong Agricultural University, Tai'an China \\ 2. Shandong Provincial Key Laboratory of Horticultural Machineries and Equipments \\ Shandong Agricultural University, Tai'an China

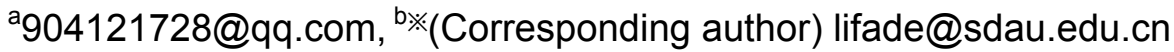

\begin{abstract}
Keywords: intelligent drawer; speech recognition; embedded microprocessor; stepper motor; ball screw
\end{abstract}

\begin{abstract}
Aiming at the problem that surgeons can not open unsterilized drawers by themselves after hand disinfection, intelligent drawer speech recognition control system was designed. The system based on non-specific person speech recognition technology takes embedded microprocessor as the core, consists of power modules, speech recognition module, stepper motor driver module and action execution system. Key words list and distance of recognition is set in program as needed, then speech recognition control system tests whether the speech of non-specific person accords with the given key words, and generates pulse width modulation signal to control stepper motor, then ball screw convert stepper motor's rotary motion into linear motion to control drawer's open and close. In order to ensure system performance, the corresponding experiments were did. Experiment results showed that speech recognition rate of system in the indoor environment can reach $90 \%$ or more, and it in the outdoor environment also can reach $81.7 \%$. System stability is better in the quiet, and control system could do correct response when key words are said two times at the most. And it has declined in the noisy, and in order to make control system do correct response, some control orders need to be said three times or more. System real-time response can be guaranteed in the quiet, and response time is less than $2 \mathrm{~s}$, it need more time in the noisy.
\end{abstract}

\section{Introduction}

Language is the most important, effective, commonly used and convenient form of communication, so it is easy to think of the idea that traditional way of man-machine communication can be replaced by natural language [1].

In general, communication of language between man and machine is roughly divided into two cases: the first case is that a machine speech, human listen. This is speech synthesis technology. The second case is that human speech, machine obedient. This is artificial recognition and understanding of speech. In the implementation and application of technology, speech recognition technology is more complex and more promising than the speech synthesis technology.

The introduction of Linear Predictive Coding technology make the feature extraction technology of speech recognition produces tremendous progress. Then the establishment of Vector Quantization and Hidden Markov Model theory realized isolated speech recognition system based on Linear Prediction Cestrum and Dynamic Time Warping technology. Successful application of Hidden Markov Model and Artificial Neural Network in speech recognition enables speech recognition gain further development[2]. Because of the rapid development and wide application of speech recognition technology, furniture controlled by speech recognition become an important development direction of smart furniture design. 


\section{Basic Theory of Speech Recognition[3]}

As figure 1, speech recognition comprises two phases: training and recognition. Whether training or recognition, all of the input speech should be pre-processed and its features will be extracted. During the training phase, user inputs some training speech, then the feature vector parameters will be obtained after preprocessing and extracting speech's feature, so that it could achieve the purpose of establishing reference model library of training speech through establishing feature model. During the recognition phase, the feature vector parameters of input speech compares with reference models in similarity measurement, then the highest similarity of input feature vector will export as a recognition result. In this way, the purpose of speech recognition is realized eventually.

\section{System Design}

Intelligent drawer control system is based on non-specific person speech recognition technology, and we realize the design of the control system from hardware and software through systematic analysis on practical application and various types of smart home system in existing literature.

The system mainly consists of speech recognition control system, action execution system and power module. And system structure sketch is as figure 2.

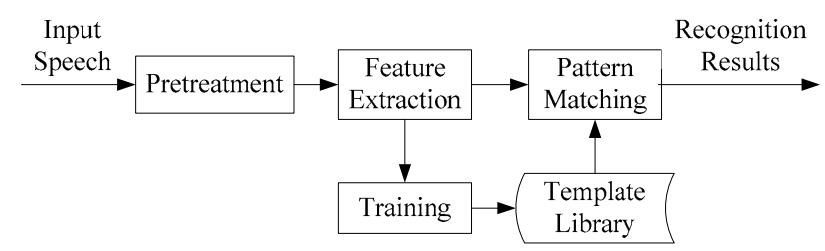

Figure 1. Speech recognition schematics

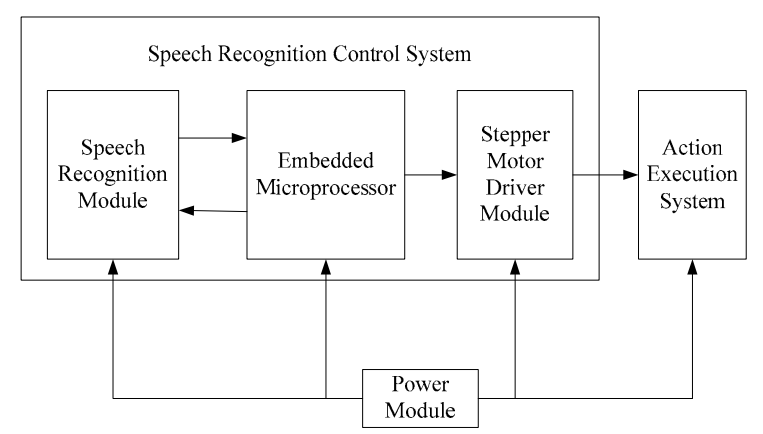

Figure 2. System structure sketch

\section{A. Design of Speech Recognition Control System}

Speech recognition control system takes embedded microprocessor as the core, and its peripheral consists of speech recognition module, and stepper motor driver module. And they are integrated in a printed circuit board, hardware schematics are as figure 3.

STM32F103C8T6 based on the ARM Cortex-M processor is designed to offer new degrees of freedom to MCU users. It offers a 32-bit product range that combines very high performance, realtime capabilities, digital signal processing, and low-power, low-voltage operation, while maintaining full integration and ease of development[4]. So it is taken as the embedded microprocessor.

Speech recognition module consists of LD3320 chip and some related peripheral circuits. LD3320 is a speech recognition chip based on Speaker Independent Automatic Speech Recognition, it integrates speech recognition processor and some external circuitry, including analog-to-digital converter, digital-to-analog converter, microphone jack and the sound output interface. And it could achieve speech recognition features without any auxiliary chip, if it is integrated directly into existing products[5]. 
The system choose ULN2068 as the stepper motor driver chip. And ULN2068 chip amplifies the pulse width modulation signal generated by the I/O pins of STM32 chip, then controls stepper motor forward and reverse through signal line.

B. Design of Action Execution System

Figure 4 shows that action execution system comprises a stepper motor, a slider and a ball screw. Ball screw and stepper motor connect together by sleeve, and slider is fixed on the screw nut of ball screw. The function of ball screw is to convert rotary motion into linear motion, so if slider is fixed on the bottom of a drawer, we could achieve drawer's open and close through stepper motor's forward and reverse.

C. Power Module Design

Power module mainly consists of switching power supply and low dropout voltage regulator. Switching power supply converts alternating voltage $220 \mathrm{~V}$ into direct voltage $5 \mathrm{~V}$ to supply power to ULN2068 chip and stepper motor. Then low dropout voltage regulator converts direct voltage $5 \mathrm{~V}$ into direct voltage $3.3 \mathrm{~V}$ to supply power to STM32 chip and LD3320 chip. So intelligent drawer control system can work stably.

D. Software Design

Figure 5 as following is programming flowchart. Speech recognition module converts the received speech signal into digital signal, then communicates with STM32 through serial peripheral interface. Then STM32 determine whether the received speech signal matches with the given key words.

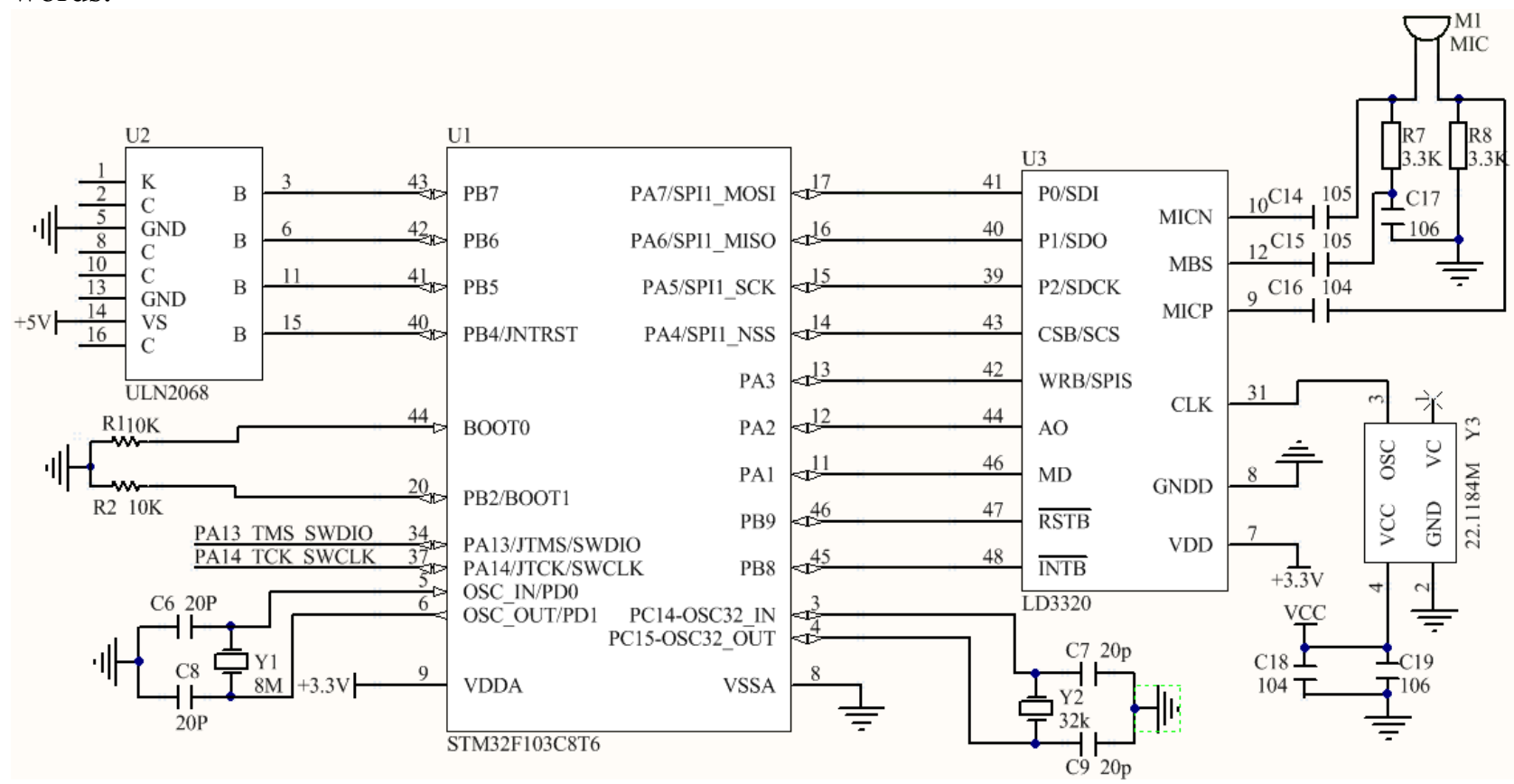

Figure 3. Hardware schematics of speech recognition control system

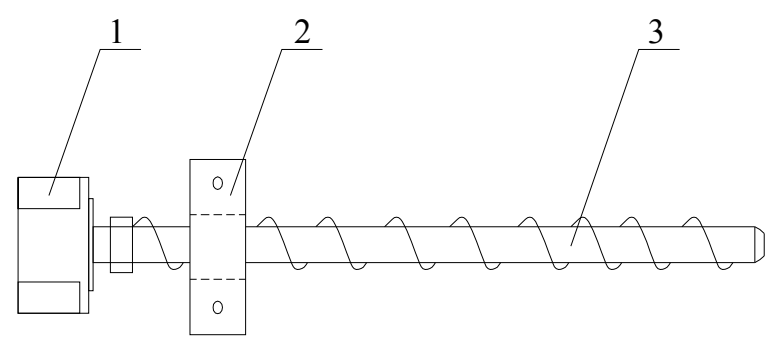

1. Stepper motor, 2. Slider, 3. Ball screw

Figure 4. Structure sketch of action execution system 


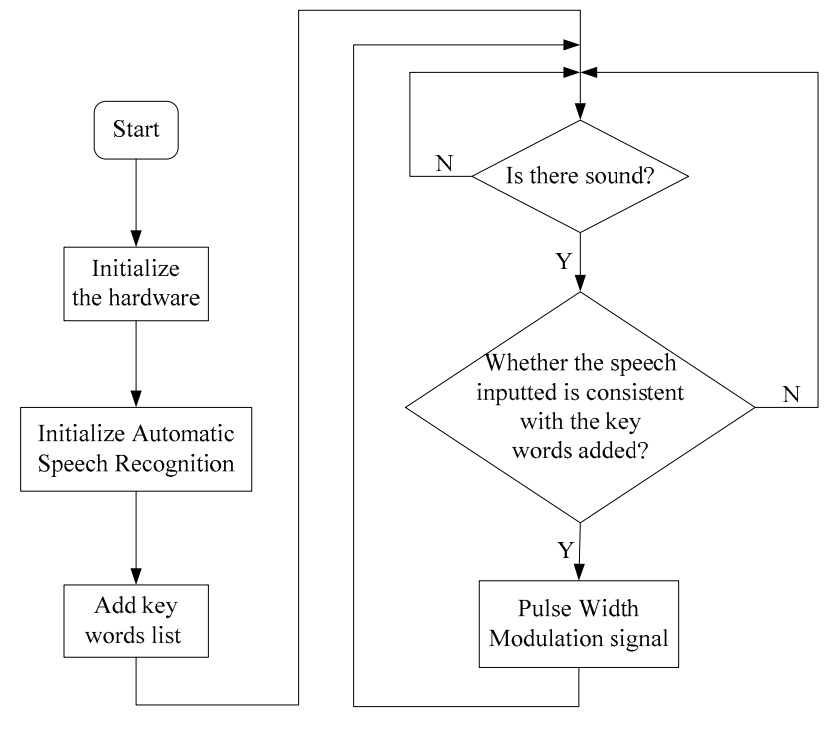

Figure 5. Programming flowchart

If it matches, the $\mathrm{I} / \mathrm{O}$ pins of the embedded microprocessor would generate corresponding pulse width modulation signal to drive stepper motor rotation. If not, there will be no action and continue to wait for the speech's generation.

The key words list and distance of recognition can be set as needed in the program, and some different words could be set to control the same action.

\section{Performance Experiment}

In order to ensure speech recognition rate, real-time response and stability of the system, the corresponding experiments were did, and experiments environment was in quiet indoor and noisy outdoor. There were three persons who did ten experiments for each of six key words respectively, and person $\mathrm{A}$ is female, person $\mathrm{B}$ and person $\mathrm{C}$ are males[6], then the number of successful identification was recorded.

The experiment results are listed in table 1. And it shows that speech recognition rate of system in the indoor environment can reach $90 \%$ or more, and speech recognition rate of system in the outdoor environment also can reach $81.7 \%$. Because female pronunciation is more standard, speech recognition rate of system for female is always higher than male. So not only the environment but also the non-specific person's pronunciation can influence system's speech recognition rate.

Table 1 also shows that in the indoor, key words are said two times at the most, the control system can do correct response. But in the outdoor, system stability has declined, and in order to make control system do correct response, some control orders need to be said three times or more. So system stability in the quiet is better than it in the noisy.

We recorded the response time from speech to action during the experiments. And we also found out that system real-time response can be guaranteed in the quiet, and response time is less than $2 \mathrm{~s}$, it need more time in the noisy environment.

Overall, the speech recognition rate, real-time response, and system stability can match the design requirements perfectly whether in quiet or noisy environment. 
TABLE I. SPEECH RECOGNITION PERFORMANCE STATISTICS

\begin{tabular}{|c|c|c|c|c|c|c|}
\hline \multirow{3}{*}{ Statement } & \multicolumn{6}{|c|}{ Environment } \\
\hline & \multicolumn{3}{|c|}{ Indoor (quiet) } & \multicolumn{3}{|c|}{ Outdoor (noisy) } \\
\hline & Person $A$ & $\begin{array}{c}\text { Person } \\
B\end{array}$ & $\begin{array}{c}\text { Person } \\
C\end{array}$ & $\begin{array}{c}\text { Person } \\
A\end{array}$ & $\begin{array}{c}\text { Person } \\
\text { B }\end{array}$ & $\begin{array}{c}\text { Person } \\
C\end{array}$ \\
\hline Go ahead & 10 & 9 & 10 & 10 & 8 & 10 \\
\hline Get back & 9 & 9 & 8 & 9 & 9 & 8 \\
\hline Start action & 10 & 9 & 9 & 8 & 8 & 8 \\
\hline Finish action & 10 & 10 & 10 & 9 & 9 & 9 \\
\hline Open the fist drawer & 9 & 9 & 8 & 7 & 8 & 6 \\
\hline Close the first drawer & 9 & 10 & 9 & 8 & 8 & 8 \\
\hline Recognition rate $/(\%)$ & 95 & 93.3 & 90 & 85 & 83.3 & 81.7 \\
\hline
\end{tabular}

\section{Summary}

Language is the primary means of information transmission for human, and speech recognition technology is one of the most popular and most promising information technology in the world[7]. Speech recognition technology as human interface device has the advantages of simple, fast and flexible, so it will play a huge role in military, civilian and commercial areas in the future.

A large number of experiments show that intelligent drawer speech recognition control system is stable, speech recognition is high, noise immunity ability is strong, and it's structure is simple. And the system liberates people's hands, and makes people open and close drawer on specific speech only, which solve the problem that surgeons can not open unsterilized drawers by themselves after hand disinfection.

\section{Acknowledgment}

First, thanks for the opportunity provided by sponsors to communication and learning. And thanks for good experiment environment provided by professor $\mathrm{Fa}-\mathrm{de} \mathrm{Li}$, and thanks for the guidance of professor Yinfa Yan.

\section{References}

[1] HE Xiang-zhi, "The Research and Development of Speech Recognition," Computer and Modernization, vol. 3, March 2005, pp. 3-6.

[2] Norio Tamaki, Shinji Matsuoka, and Keiji Harada, "Recent application and development in speech recognition technologics," NTT Review, vol. 16, April 1994, pp. 66-75.

[3] JIN Xin, TIAN Ben, and QUE Da-shun, "Design of Voice-Controlled System Based on LD3320," Computer and Information Technology, vol. 19, December 2001, pp. 22-25.

[4] STM32F103x8/ B Enhanced series data sheet [EB/OL]. [2009-4] http://www.stmicroelectronics.com.cn/stonline/mcu/MCU_Pages.htm

[5] ICRoute. LD332X Data Sheet [EB/OL]. [2010-3-10] http://www.icroute.com/index.html

[6] Su Peng, Zhou Fenyu, and Chen Lei, "Embedded Speech Recognition Module Based on STM32," NEW PRODUCT \& TECH, vol. 2, 2011, pp. 42-45.

[7] Yoo Oh, Jae Yoon, K. Oka, and Ji Park, "A name recognition based call-and-come service for home robots," IEEE Transactions on Consumer, vol. 54, May 2008, pp. 247-253. doi: 10.1109/TCE.2008.4560082. 